\title{
ON THE STEENROD OPERATIONS IN CYCLIC COHOMOLOGY
}

\author{
MOHAMED ELHAMDADI and YASIEN GH. GOUDA
}

Received 12 September 2002

\begin{abstract}
For a commutative Hopf algebra $A$ over $\mathbb{Z} / p$, where $p$ is a prime integer, we define the Steenrod operations $P^{i}$ in cyclic cohomology of $A$ using a tensor product of a free resolution of the symmetric group $S_{n}$ and the standard resolution of the algebra $A$ over the cyclic category according to Loday (1992). We also compute some of these operations.
\end{abstract}

2000 Mathematics Subject Classification: 55N20, 55S20, 13D03, 16E40.

1. Introduction. For any prime $p$, the $\bmod p$ Steenrod algebra $A(p)$ is the graded associative algebra generated by the $\bmod p$ stable operations $P^{i}$ of degree $2 i(p-1)$ in the ordinary cohomology theory. When $p=2$, it is generated by the Steenrod squares $S q^{i}(i \geq 1)$ subject to the Adem relations. The operations $P^{i}$ and $S q^{i}$ increase degree, respectively, by $2 i(p-1)$ and $i$; in other words,

$$
\begin{aligned}
P^{i}: H^{q}(-, \mathbb{Z} / p) & \rightarrow H^{q+2 i(p-1)}(-, \mathbb{Z} / p), \\
S q^{i}: H^{q}(-, \mathbb{Z} / p) & \longrightarrow H^{q+i}(-, \mathbb{Z} / p) .
\end{aligned}
$$

In [4], Epstein introduced the Steenrod operations into derived functors and obtained as a special case the Steenrod operations in the cohomology of groups and in the cohomology of a space with coefficients in sheaves (see also [15]). Other operations like Adams' were studied in [5, 11]. The $S$ - and $\lambda$-operations in cyclic homology have been defined and studied in [2]. Some special operations (dot product, bracket) on Hochschild complex that induce a structure of graded algebra on the cohomology have been considered in [16]. Steenrod operations on the Hochschild homology have been studied in [13]. There are also operations in $K$-theory, for instance [8], and $\lambda$-operations in orthogonal $K$ theory [3]. Many applications of the Steenrod algebra have been made: in 1958, Adams [1] used them to compute the stable homotopy groups of spheres and in the same year Milnor [12] proved that the Steenrod algebra and its dual have structures of Hopf algebras.

In this paper, we define the Steenrod operations in cyclic cohomology of a commutative Hopf algebra and obtain some calculations. 
2. Steenrod operations on cyclic cohomology. Let $k$ be a commutative ring with unit, $A$ a commutative $k$-Hopf algebra, and $\mathscr{b}$ a cyclic category (see [10, page 202]). We will denote the $k$-algebra over $\mathscr{b}$ by $k[\mathscr{b}]$ and the cyclic category over $A$ by $A^{\mathscr{C}}$ (see [10]). We define an $A^{\mathscr{C}}$-structure of cocommutative coalgebra by the formula

$$
A^{\mathscr{C}} \stackrel{\nabla}{\longrightarrow} A \otimes A \stackrel{f}{\longrightarrow} A^{\mathscr{C}} \otimes A^{\mathscr{C}}
$$

where $\nabla$ is $k[\mathscr{C}]$-homomorphism and $f$ is given by

$$
\begin{aligned}
f\left(\left(a_{0} \otimes b_{0}\right) \otimes\left(a_{1} \otimes b_{1}\right) \otimes \cdots \otimes\left(a_{n} \otimes b_{n}\right)\right) \\
=\left(a_{0} \otimes a_{1} \otimes \cdots \otimes a_{n}\right) \otimes\left(b_{0} \otimes b_{1} \otimes \cdots \otimes b_{n}\right) .
\end{aligned}
$$

Suppose that $\nabla^{\mathscr{C}}=f \circ \nabla$ gives the cocommutative comultiplication in $A^{\mathscr{C}} \otimes_{k} A^{\mathscr{C}}$, that is, $T \circ \nabla^{\mathscr{C}}=\nabla^{\mathscr{C}}$, where $T$ is the twisting map $T(a \otimes b)=b \otimes a$. We have, for $x$ in $k[\mathfrak{G}]$,

$$
\begin{aligned}
f\left(x\left[\left(a_{0} \otimes b_{0}\right) \otimes \cdots \otimes\left(a_{n} \otimes b_{n}\right)\right]\right) & =x\left(a_{0} \otimes \cdots \otimes a_{n}\right) \otimes x\left(b_{0} \otimes \cdots \otimes b_{n}\right) \\
& =x\left[\left(a_{0} \otimes b_{0}\right) \otimes \cdots \otimes\left(a_{n} \otimes b_{n}\right)\right] \\
& =x f\left(\left(a_{0} \otimes b_{0}\right) \otimes \cdots \otimes\left(a_{n} \otimes b_{n}\right)\right) .
\end{aligned}
$$

The comultiplication $\nabla^{\mathscr{C}}$ becomes a $k[\mathfrak{G}]$-module homomorphism.

2.1. The normalized bar construction. Let $J k[\mathscr{C}]$ be the cokernel of the $k$ map $k \rightarrow k[\mathscr{C}]$. The normalized bar construction of the triple $L=\left(A^{\mathscr{C}}, k[\mathscr{C}], k^{\mathscr{C}}\right)$ is defined to be the graded $k$-module $B(L)$ with

$$
B_{m}(L)=A^{\mathscr{C}} \otimes_{k[\mathscr{C}]} T^{m}(J k[\mathscr{C}]) \otimes_{k[\mathscr{C}]} k^{\mathscr{C}},
$$

where $T^{m}(J k[\mathscr{C}])$ is the tensor algebra in degree $m$. As $k$-module $B_{m}(L)$ is spanned by elements written as $a\left[g_{1}|\cdots| g_{m}\right] u$, where $a$ is in $A^{\mathscr{C}}, g_{i}$ belongs to $k[\mathscr{C}]$, and $u$ is an element of $k^{\mathscr{C}}$. The differential $d_{m}: B_{m}(L) \rightarrow B_{m-1}(L)$ is given by

$$
\begin{aligned}
d_{m}\left(a\left[g_{1}|\cdots| g_{m}\right] u\right)= & a g_{1}\left[g_{2}|\cdots| g_{m}\right] u \\
& +\sum_{i=1}^{m-1}(-1)^{i} a\left[g_{1}|\cdots| g_{i-1}\left|g_{i} g_{i+1}\right| g_{i+2}|\cdots| g_{m}\right] u \\
& +(-1)^{m} a\left[g_{1}|\cdots| g_{m-1}\right] g_{m} u .
\end{aligned}
$$

The elements are normalized in the sense that $f\left(\left[g_{1}|\cdots| g_{m}\right] u\right)=0$ and $f(a[\cdot] u)=0$, where $a[\cdot] u$ are elements of $B_{0}$.

We define also, for the triple $T=\left(k[\mathscr{b}], k[\Delta \mathscr{C}], k^{\mathscr{C}}\right)$, the maps $d$ and $f$ in the same manner. Note that for $T$, the differential $d$ is a left $k[\mathscr{C}]$-module homomorphism and $d s+s d=1-\sigma f$, where the morphisms $\sigma: k^{\mathscr{C}} \rightarrow B(T)$ 
and $s: B_{m}(T) \rightarrow B_{m-1}(T)$ are given by the formulas $\sigma(u)=[\cdot] u \otimes_{k}[\cdot]$ and $s\left(g\left[g_{1}|\cdots| g_{m}\right] u\right)=g\left[g_{1}|\cdots| g_{m}\right] u$. It is clear that the differential $d$ in the complex $B(L)$ is equal to $1 \otimes_{k[\bullet]} d$. We have the equality

$$
\operatorname{Hom}_{k[\Delta \mathscr{C}]}\left(B(T),\left(A^{\mathscr{C}}\right)^{*}\right)=(B(L))^{*}=\operatorname{Hom}_{k[\Delta \mathscr{C}]}\left(B\left(A^{\mathscr{C}}\right), k[\mathscr{C}], k[\mathscr{C}]^{\mathscr{C}},(k)^{*}\right) .
$$

We then have (see [10, page 214]),

$$
H C^{n}(A)=\operatorname{Ext}_{k[\mathscr{C}]}^{n}\left(A^{\mathscr{C}},\left(k^{\mathscr{C}}\right)^{*}\right)=H^{n}\left((B(L))^{*}\right) .
$$

Given a triple $L$ and considering the product $\perp: B(L \otimes L) \rightarrow B(L) \otimes B(L)$, we define on $B(L)$ a structure of coassociative coalgebra by means of comultiplication $\tilde{\nabla}=\perp B\left(\nabla^{\mathscr{C}}, \nabla_{k[\mathscr{G}]}, \nabla_{k^{\mathscr{C}}}\right): B(L \otimes L) \rightarrow B(L) \otimes B(L)$ and on $B(L)^{*}$ the following multiplication as a composite map:

$$
B(L)^{*} \otimes B(L)^{*} \longrightarrow(B(L) \otimes B(L))^{*} \stackrel{(\tilde{\nabla})^{*}}{\longrightarrow} B(L)^{*} .
$$

We have the following lemma which can be easily proved by ordinary techniques of homological algebra (see [15]).

LEMMA 2.1. Let $\mu$ be an arbitrary subgroup of the symmetric group $S_{n}$ and $W$ the free resolution of $k$ as $k[\mu]$-module with a generator $e_{0}$. Then there is a graded $k[\mu]$-complex with the following properties:

(a) $\Delta(w \otimes b)=0$ for $b \in B_{0}(L)$ and $w \in W_{i}, i>0$;

(b) $\Delta\left(e_{0} \otimes b\right)=\tilde{\nabla}^{\otimes r}(b)$ for $b \in B(L)$ and $\tilde{\nabla}^{\otimes r}: B(L) \rightarrow B(L)^{\otimes r}$;

(c) the map $\Delta: B(L) \rightarrow B(L)^{\otimes r}$ is a left $k[\mathfrak{C}]$-module, homomorphism, where $k[\bullet]$ acts on $W \otimes B(L)$ by $u(w \otimes b)=w \otimes u b$;

(d) $\Delta\left(W_{i} \otimes B_{m}(L)\right)=0$ when $i>(r-1) m$.

Furthermore, there exists a $k[\mu]$-homotopy between any two homomorphisms $\Delta$ with the same properties.

Now define a $k[\mu]$-homomorphism $\theta: W \otimes\left((B(L))^{*}\right)^{\otimes r} \rightarrow(B(L))^{*}$ with $\theta(w \otimes$ $x)(m)=B(x) \Delta(w \otimes x), w \in W, x \in\left((B(L))^{*}\right)^{\otimes r}, m \in B(L)$, and $B:\left((B(L))^{*}\right)^{\otimes r}$ $\rightarrow\left((B(L))^{\otimes r}\right)^{*}$ a trivial homomorphism.

2.2. Operations. In the above lemma, let $\mu=\mathbb{Z} / p$ and $k=\mathbb{Z} / p$, where $p$ is a prime integer. Consider the $k[\mathbb{Z} / p]$-free resolution $W$ with $W_{i}, i \geq 0$, generated by $e_{i}$. For $i<0$, consider $W_{i}:=W_{-i}$ as a free $k[\mathbb{Z} / p]$-module with a generator $e_{-i}$. Now we define, for $i \geq 0$, the homomorphism

$$
\begin{gathered}
R_{i}: H^{q}\left(B(L)^{*}\right) \longrightarrow H^{p q-i}\left(B(L)^{*}\right), \\
x \longmapsto R_{i}(x)=\theta^{*}\left(e_{-i} \otimes x^{p}\right) .
\end{gathered}
$$

We extend the definition of this homomorphism to the negative $i$ by $R_{i}=0$. The Steenrod operations $P^{i}$ are defined in terms of the $R_{j}$ in the following manner. 
(a) For $p=2, P^{i}:=R_{q-i}: H^{q}\left(B(L)^{*}\right) \rightarrow H^{q+i}\left(B(L)^{*}\right)$.

(b) For $p$ a prime integer greater than $2, P^{i}: H C^{n}(A) \rightarrow H C^{n+2 i(p-1)}(A)$ is given by $P^{i}(x)=(-1)^{i+j}((p-1) / 2 !)^{\epsilon} R_{(n-2 i)(p-1)}(x)$, where $n=2 j-\epsilon$, $\epsilon=0$ or 1 , and $x \in H C^{n}(A)$, and $\beta P^{i}: H C^{n}(A) \rightarrow H C^{n+2 i(p-1)}(A)$ is given by $\beta P^{i}(x)=(-1)^{i+j}((p-1) / 2 !)^{\epsilon} R_{(n-2 i)(p-1)-1}(x)$.

DEFINITION 2.2. Let $A$ be a commutative $k$-Hopf algebra where $k=\mathbb{Z} / p$. The Steenrod maps are the homomorphisms $P^{i}: H C^{n}(A) \rightarrow H C^{n+i}(A)$, when $p=2$, and $P^{i}: H C^{n}(A) \rightarrow H C^{n+2 i(p-1)}(A)$, when $p>2$. In this case, $\beta P^{i}: H C^{n}(A) \rightarrow$ $H C^{n+2 i(p-1)}(A)$.

We then have the following properties of these operators.

THEOREM 2.3. (a) When $p=2$ and $n<i$ or $n<2 i<2 n, P^{i}: H C^{n}(A) \rightarrow$ $H C^{n+i}(A)$ is equal to zero. Also, $\beta P^{i}: H C^{n}(A) \rightarrow H C^{n+2 i(p-1)}(A)$ is zero when $n<2 i$.

(b) When $i=n$ and $p=2, P^{i}(x)=x^{2}$.

(c) The Steenrod maps satisfy $P^{n}=\sum_{i=0}^{n} P^{i} \otimes P^{n-i}$ and $\beta P^{n}=\sum_{i=0}^{n} \beta P^{i} \otimes$ $P^{n-i}+P^{i} \otimes \beta P^{n-i}$.

(d) The operations $P^{n}$ and $\beta P^{n}$ satisfy the following Adem relations:

(i) for $p \geq 2$ and $m<p n$,

$\beta^{\gamma} P^{m} P^{n}=\sum_{i}(-1)^{m+i}\left(\begin{array}{c}m-p i+(p-1)(n-m+i-1) \\ m-p i\end{array}\right) \beta^{\gamma} P^{m+n-i} P^{i}$,

where $(\cdot)$ is the binomial coefficient, $\gamma=0$ or 1 , when $p=2$, and $\gamma=1$, when $p>2$,

(ii) for $p>2$, $p n \geq m$, and $\gamma=0$ or 1 ,

$$
\begin{aligned}
\beta^{\gamma} P^{m} P^{n}= & (1-\gamma) \sum_{i}(-1)^{m+i}\left(\begin{array}{c}
m-p i+(p-1)(n-m+i-1) \\
m-p i
\end{array}\right)(\beta P)^{m+n-i} P^{i} \\
& -\sum_{i}(-1)^{m+i}\left(\begin{array}{c}
m-p i+(p-1)(n-m+i-1) \\
m-p i
\end{array}\right) \beta^{\gamma} P^{m+n-i}(\beta P)^{i} .
\end{aligned}
$$

Proof. Consider the triple $C=(E, A, F)$, where $A$ is a cocommutative Hopf algebra over $\mathbb{Z} / p, E$ and $F$ are, respectively, the right and left cocommutative coalgebras over $A$. From the above discussion and considering the triple $L=\left(A^{\mathscr{C}}, k[\mathscr{C}], k^{\mathscr{C}}\right)$, then $k[\mathscr{C}], A^{\mathscr{C}}$, and $k^{\mathscr{C}}$ become, respectively, cocommutative Hopf algebra over $\mathbb{Z} / p$, and right and left cocommutative $k[\mathscr{C}]$-coalgebras, and then $H^{n}\left(B(L)^{*}\right)=H C^{n}(A)$.

REMARK 2.4. Note that if we replace the category $k[\mathscr{b}]$ by a reflexive category $k[R]$ (see $[7,9]$ ), then the Steenrod operations can be defined on the reflexive homology. 
3. Some computations of Steenrod operations. We use operads and algebra of operads to obtain some computations of the Steenrod operations on the cohomology of a Hopf algebra over $\mathbb{Z} / p$. Let $H^{*}$ be the cohomology of the Hopf algebra $A$ and consider the Steenrod operations

$$
P^{i}: H C^{n}\left(S, H^{*}\right) \rightarrow H C^{n+i}\left(S, H^{*}\right),
$$

where the algebra $S$ over operad is the $S_{w}$-algebra structure over $H^{*}$ and $S_{w}=$ $\left\{S_{w}(j)\right\}_{j}$ is the cyclic operad generated by elements $u_{i} \in S_{w}(2)$ and $\pi_{i} \in S_{w}(i+$ 2) (see [6]).

Proposition 3.1. There is an $S_{w}$-algebra over $H^{*}$ generated by an element $h_{0}$ of dimension one such that $\pi_{i}\left(h_{0}, h_{1}, \ldots, h_{i+1}\right)=0$, where $h_{i}$ are given inductively by $h_{i+1}=h_{i} P^{1} h_{i}$.

LEMMA 3.2 [14]. Let $X$ be a simplicial complex, $C X$ the free commutative coalgebra generated by $X, A$ a Steenrod algebra where $P^{0}=1, A\left[H^{*}(X)\right]$ a free unstable A-module generated by $H^{*}(X)$, and $S\left\{A\left[H^{*}(X)\right]\right\}$ a commutative algebra generated by $A\left[H^{*}(X)\right]$ with multiplication given by $x \cdot x=x \cup x$. Then $H^{*}(C X) \cong S\left\{A\left[H^{*}(X)\right]\right\}$.

LEMмA 3.3. There exists a chain equivalence $B\left(S A, H^{*}\right) \cong B\left(A, B\left(S, H^{*}\right)\right)$.

Proof (sketch). Let $Y_{*}$ denote the cohomology of $\left(S A, H^{*}\right)$. We then have the complex

$$
B\left(A, Y_{*}\right): \cdots \rightarrow A^{2} Y_{*} \rightarrow A Y_{*} \longrightarrow Y_{*}
$$

with the cohomology given by

$$
H^{n}\left(B\left(A, Y_{*}\right)\right)= \begin{cases}0 & n>1, \\ H^{i} & n=0 .\end{cases}
$$

The nontrivial cohomology group $H^{i}$ is generated by elements $\xi_{i}$, and $Y_{*}$ is clearly a free unstable $A$-module with generator $\xi_{i}$ and $A Y_{*}=H^{n}\left(B\left(S, H^{*}\right)\right)$ with generator $\xi_{i+1}$.

Proof of Proposition 3.1. Consider the diagram

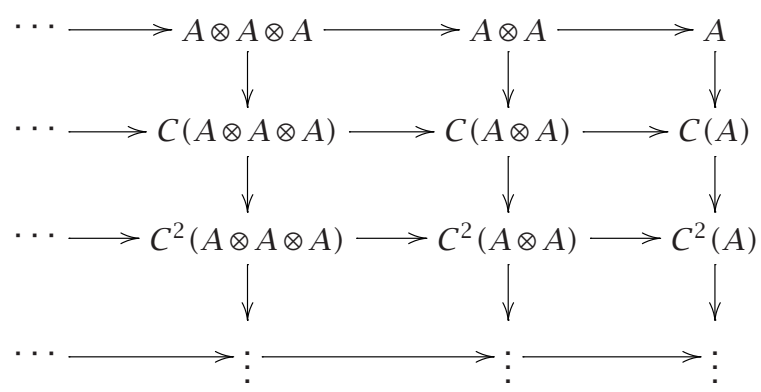


where $A$ is the Hopf algebra over $\mathbb{Z} / p$ and $C(A)$ is a free cocommutative coalgebra generated by $A$. The cohomology of the first row is by definition $H^{*}$. Consider the $B$ construction $B\left(S, H^{*}\right)$, where the differential is defined as $S_{w}$-algebra structure on $H^{*}$. The zero-dimensional cohomology of this $B$ construction contains the indecomposable elements in $H^{*}$ and also the elements

$$
\begin{gathered}
h_{1}=h_{0} P^{1} h_{0}, \quad h_{2}=h_{1} P^{1} h_{1}, \ldots, h_{i}=h_{i-1} P^{1} h_{i-1}, \\
h_{i}^{2}=h_{i} P^{0} h_{i}, \quad h_{i}^{2^{2}}=h_{i}^{2} P^{0} h_{i}^{2}, \ldots, h_{i}^{2^{k}}=h_{i}^{2^{k-1}} P^{0} h_{i}^{2^{k-1}}, \quad \text { where } h_{i}^{2^{k}} \in A^{2^{k}} .
\end{gathered}
$$

Note that these elements are also indecomposable. The one-dimensional cohomology of $B\left(S, H^{*}\right)$ is a free unstable $A$-module with one generator $\xi_{2}=$ $h_{0} h_{1} \in S^{1} H^{*}$, which means that $h_{0} h_{1}$ is acyclic $\left(\pi_{i}\left(h_{0}, h_{1}\right)=0\right)$. Consequently, the $i$-dimensional cohomology has one generator $\xi_{i+1} \in S^{i} H^{*}$, where $\xi_{i+1}=$ $\left(h_{0} \cdots h_{i+1}\right)$. Hence $\pi_{i}\left(h_{0} \cdots h_{i+1}\right)=0$.

CONSEQUENCES. From the above discussion, we conclude that the indecomposable elements in $H^{*}$ are $h^{2} \in A^{2}$ and multiplication between these elements is given by the Cartan formula

$$
(X Y) P^{n}(X Y)=\sum_{i=0}^{i=n}\left(X P^{i} X\right)\left(Y P^{n-i} Y\right) .
$$

(a) Using the operation $P^{2}$ with $h_{0} h_{1}=0$, we obtain $h_{i} h_{i+1}=0$.

(b) Taking the operation $P^{1}$ and $h_{i} h_{i+1}=0$, we obtain $h_{i} h_{i+k+2}=0$ for any nonnegative integer $k$.

(c) If we use the operation $P^{3}$, we get the relations $h_{i} h_{i+k+2}=0$ for any nonnegative integer $k$.

\section{REFERENCES}

[1] J. F. Adams, On the structure and applications of the Steenrod algebra, Comment. Math. Helv. 32 (1958), 180-214.

[2] F. Cohen, The homology of $\xi_{n+1}$-space, $n \geq 0$, The Homology of Iterated Loop Spaces, Lect. Notes in Math., vol. 533, Springer-Verlag, 1976, pp. 207351.

[3] M. Elhamdadi, A note on $\lambda$-operations in orthogonal $K$-theory, Proc. Amer. Math. Soc. 128 (2000), no. 1, 1-4.

[4] D. B. A. Epstein, Steenrod operations in homological algebra, Invent. Math. 1 (1966), 152-208.

[5] B. L. Fĕgin and B. L. Tsygan, Additive K-theory, K-Theory, Arithmetic and Geometry (Moscow, 1984-1986), Lecture Notes in Math., vol. 1289, Springer, Berlin, 1987, pp. 67-209.

[6] E. Getzler and M. M. Kapranov, Modular operads, Compositio Math. 110 (1998), no. 1, 65-126.

[7] Y. Gh. Gouda, On the cyclic and dihedral cohomology of Banach spaces, Publ. Math. Debrecen 51 (1997), no. 1-2, 67-80. 
[8] D. R. Grayson, Adams operations on higher K-theory, K-Theory 6 (1992), no. 2, 97-111.

[9] R. L. Krasauskas, S. V. Lapin, and Yu. P. Solov'ev, Dihedral homology and cohomology. Basic concepts and constructions, Mat. Sb. (N.S.) 133(175) (1987), no. 1, 25-48, (Russian).

[10] J.-L. Loday, Cyclic Homology, Grundlehren der mathematischen Wissenschaften, vol. 301, Springer-Verlag, Berlin, 1992.

[11] J.-L. Loday and C. Procesi, Cyclic homology and lambda operations, Algebraic $K$ Theory: Connections with Geometry and Topology (Lake Louise, Alberta, 1987), NATO Adv. Sci. Inst. Ser. C Math. Phys. Sci., vol. 279, Kluwer Academic Publishers, Dordrecht, 1989, pp. 209-224.

[12] J. Milnor, The Steenrod algebra and its dual, Ann. of Math. (2) 67 (1958), no. 1, 150-171.

[13] B. Ndombol and J.-C. Thomas, Steenrod operations and Hochschild homology, preprint, 2001, http://xxx.lanl.gov/abs/math.AT/0109146.

[14] V. A. Smirnov, Homology of symmetric products, Math. Notes 49 (1991), no. 1-2, 73-80.

[15] N. E. Steenrod and D. B. A. Epstein, Cohomology Operations, Annals of Mathematics Studies, no. 50, Princeton University Press, New Jersey, 1962, lectures by N. E. Steenrod written and revised by D. B. A. Epstein.

[16] A. A. Voronov and M. Gerstenkhaber, Higher-order operations on the Hochschild complex, Funct. Anal. Appl. 29 (1995), no. 1, 1-5.

Mohamed Elhamdadi: Department of Mathematics, University of South Florida, Tampa, FL 33620, USA

E-mail address: emohamed@math.usf.edu

Yasien Gh. Gouda: Department of Mathematics, Faculty of Science, South Valley University, Aswan, Egypt

E-mail address: yasi en10@hotmai 1.com 


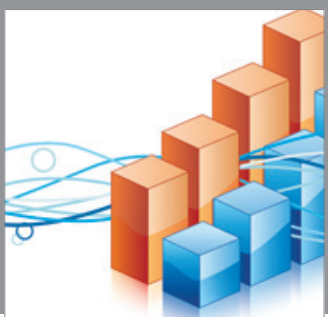

Advances in

Operations Research

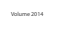

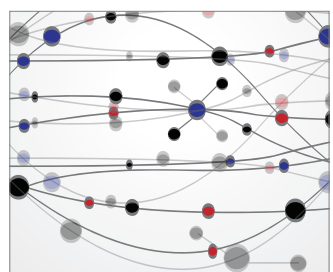

\section{The Scientific} World Journal
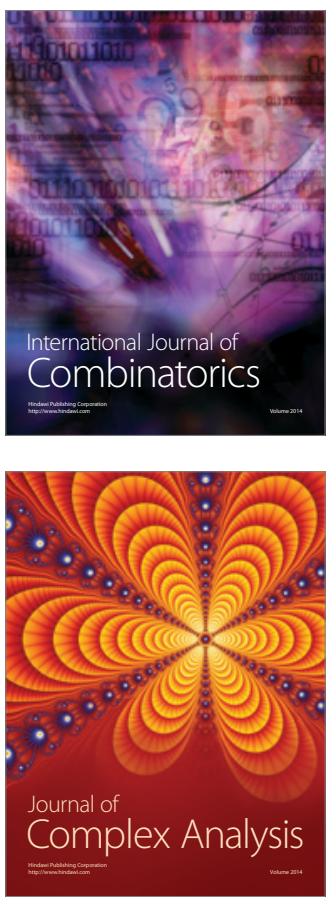

International Journal of

Mathematics and

Mathematical

Sciences
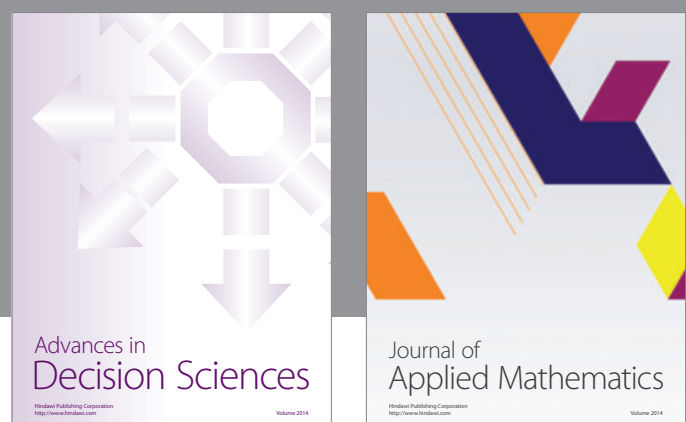

Journal of

Applied Mathematics
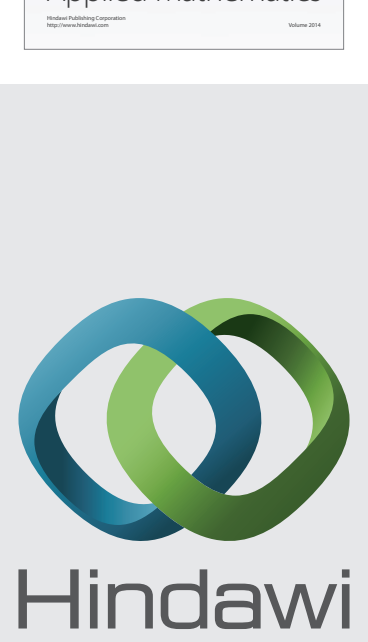

Submit your manuscripts at http://www.hindawi.com
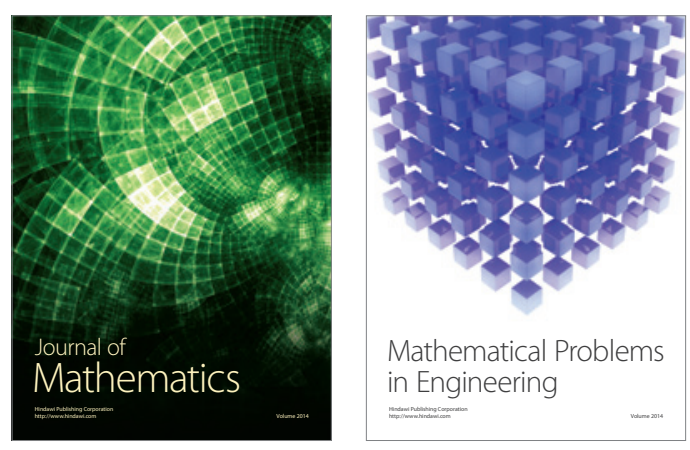

Mathematical Problems in Engineering
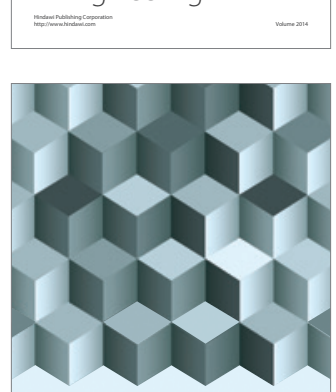

Journal of

Function Spaces
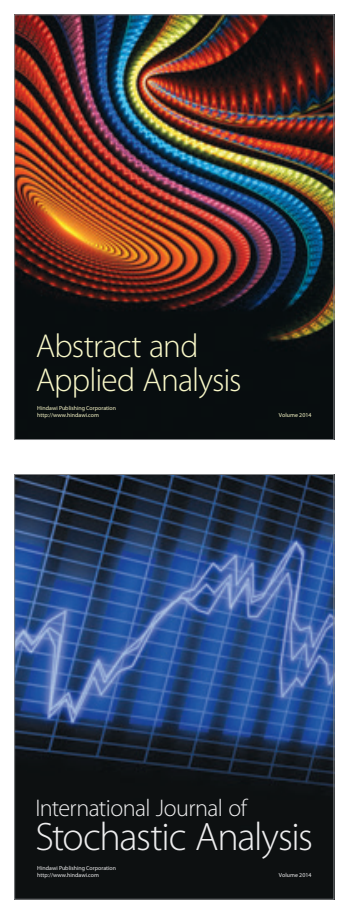

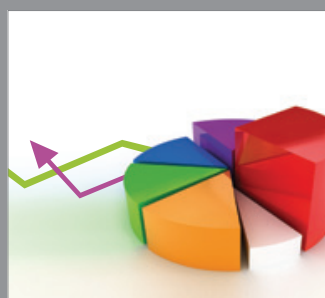

ournal of

Probability and Statistics

Promensencen
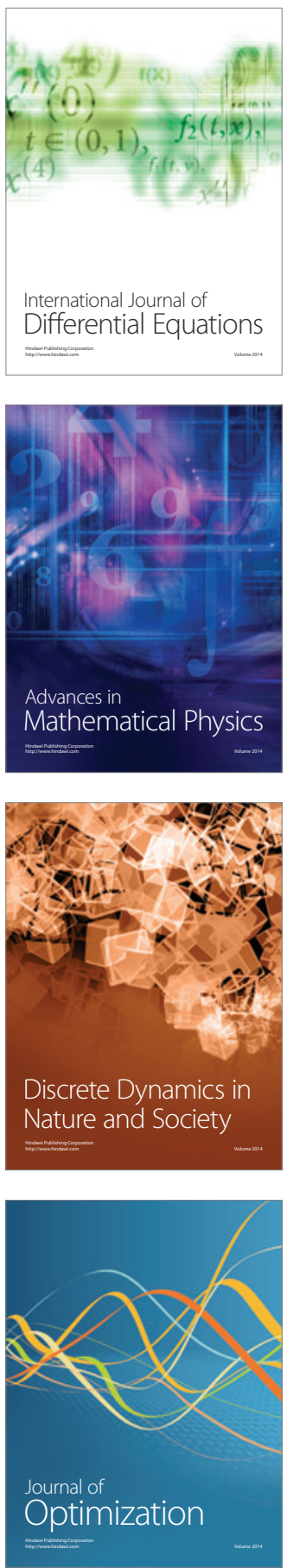